\title{
Zur Ähnlichkeit der Bilder: oder wie reden wir eigentlich über Bilder?
}

Jakob Steinbrenner, München

Schaut man sich die Debatte zwischen Ähnlichkeitstheoretikern und Zeichentheoretikern an, scheinen beide an der Rede über Bilder nur bedingt interessiert zu sein (vgl. Steinbrenner 2009). Denn weder die Umriss- oder Farbgleichheit noch die syntaktischen oder semantischen Merkmale von Bildern spielen in unserer Rede über Bilder eine wesentliche Rolle. Das - so könnte man meinen - muss den Theoretiker auch nicht weiter interessieren, insofern seine Theorie Klärung über unseren Umgang mit Bildern gibt. Aber was soll hier genauer geklärt werden und welchen Status haben die Sätze, die zur Klärung gebraucht werden? Dies ist ein dunkles Thema, auf das ich hier nicht genauer eingehen kann (vgl. Steinbrenner im Erscheinen). Als Fazit der Diskussion zwischen Ähnlichkeitstheoretikern und Zeichentheoretikern kann man meines Erachtens nichtsdestotrotz festhalten, dass der Ausdruck der Ähnlichkeit uns nur bedingt weiter hilft bei unserem Versuch zu verstehen, wie wir mit Bildern umgehen (vgl. Steinbrenner 1998), und Zeichentheorien bis heute wenige Erklärungen dazu anzubieten haben, wenn es darum geht, welche Erlebnisse und Eindrücke zentral für unseren Umgang mit Bildern sind (vgl. Lopes 1996, Kap. 1.6). Nach meiner Auffassung kommt es zu diesen Theoriedefiziten deshalb, weil die Akteure zu wenig auf Wittgensteins Diktum hören, dass an die Stelle von Erklärungen bloße Beschreibungen treten sollten. Ich möchte im Folgenden versuchen zu zeigen, dass, wenn man sich diesem Diktum Wittgensteins beugt, man zu tieferen Einsichten darüber gelangen kann, was Bilder sind. Im Idealfall sollte dies dazu führen, an und in unserer Rede über Bilder das Spezifikum von Bildern zu erkennen. Ich werde mich dabei, wie es in der gegenwärtigen Diskussion über Bilder nicht unüblich ist, im Wesentlichen auf - wie sie Dominic Lopes nennt demotische Bilder (ebd. S. 6 f.). beschränken, also gegenständliche materielle 
Bilder und gegenstandslose, metaphorische, geistige und andere Typen von Bildern erst einmal weitgehend ausklammern. Meine Herangehensweise besteht also darin, unsere Rede über Bilder genauer zu betrachten, mit dem Ziel, aus dieser Rede mehr über Bilder selbst zu erfahren.

Betrachtet man unsere Rede über Bilder, lassen sich folgende Kategorien unterscheiden:

I. Wir reden über Bilder mehr oder minder gleich wie über die dargestellten Gegenstände selbst.

Beispiel: Da hat der Obelix aber eine rote Nase.

II. Wir reden über Bilder als Repräsentationen von tatsächlichen Ereignissen, Personen etc. in der Welt.

Beispiel: Auf dem Bild ist der Herr aus Wien zu sehen, den wir im Urlaub kennengelernt haben.

III.Wir reden über Darstellungsqualitäten.

Beispiel: Auf dem Bild bist du gut getroffen.

IV. Wir reden über nicht-darstellende Qualitäten von Bildern.

Beispiel: Das Bild wurde für 100.000 Dollar versteigert.

Inwieweit die hier vorgeschlagenen Kategorien strikte Trennungen sind, ist natürlich äußert fraglich. Erinnert sei an Arthur Dantos Einwand, in dem er darauf hinweist, dass man bei Kunstwerken Form- respektive Stileigenschaften nicht strikt von ihrem Inhalt trennen kann. Sein Beispiel hierzu sind die äußerst knappen Sätze von Hemingway und die ausufernden Sätze von Proust und der unmögliche Versuch im Stile Hemingways Proust-Inhalte wiederzugeben und vice versa (ders. 1981, 196 f.). Bestritten soll auch nicht werden, dass sich diese Kategorien wiederum in Subkategorien einteilen lassen. Deutlich sollte aber im Folgenden werden, dass mit jeder Kategorie auch spezifische Verstehensleistungen einhergehen, d.h. ein Kleinkind wird vielleicht die Rede der ersten Kategorie, nicht aber diejenige der dritten beherrschen.

Ich werde mich im Folgenden im Wesentlichen auf die erste Kategorie beschränken, weil sie mir als die grundlegende in unserem Umgang bzw. Reden über Bilder erscheint. In gewissem Sinn deckt sich dies mit den 
Ansichten von Kendall Walton (1990, S. 83) wie auch John Hyman (2006, S. 189), die beide die These vertreten, dass der fiktionale Gebrauch von Bildern dem abbildenden Gebrauch von Tatsächlichem vorausgeht. So könnten wir uns zwar leicht eine Gesellschaft vorstellen, in der Bilder nur fiktional verwendet würden und nicht als Abbildungen von tatsächlichen Gegenständen; aber eine ähnliche Vorstellung bezogen auf die Sprache sei nur schwer denkbar. Das heißt, beide Autoren sind sich darin einig, dass unsere Beschäftigung nicht primär dem Zweck dient, Bilder als Repräsentationen der Welt zu betrachten, sondern dass Bilder uns erlauben, Aufschlüsse über die dargestellten Dinge selbst zu erlangen. Es geht bei Bildern also primär um den Inhalt und nur sekundär um den - wie es Oliver Scholz ausdrückte (ders. 2004, 177 ff.) - denotativen Sachbezug.

Zum besseren Verständnis dafür, was ich unter der ersten Kategorie genauer verstehe, betrachten wir folgende Beispielssätze:

1. Andy trägt eine platinfarbene Perücke.

2. Er sieht, dass Andy eine platinfarbene Perücke trägt.

3. Ich sehe, dass Andy eine platinfarbene Perücke trägt.

4. Schau auf das leuchtende Rot.

5. Schau, wie das Gold glänzt.

6. Andy ist kleiner als Divine.

7. Andy sieht größer als Divine aus.

8. Er sieht, dass Andy kleiner als Divine ist.

9. Mir erscheint Andy kleiner als Divine.

10. Divine stellt Bundys Mutter dar.

11. Hier sieht Andy aber gut aus.

12. Andy sieht wie sein Vater aus.

All diese Sätze und natürlich viele mehr können wir vor Bildern wie gegebenenfalls auch vor den von ihnen dargestellten Personen, Gegenständen oder Szenen wahrheitsgemäß oder korrekterweise äußern.

Meine bereits erwähnte These lautet daher, dass die ganze Rede über die Ähnlichkeit bei Bildern sich letztlich darauf zurückführen lässt, dass wir Sätze der obigen Art wahrheitsgemäß gleichermaßen vor Bildern wie vor den Gegenständen selbst äußern können.

Ähnliche Ansichten, wenn auch mit unterschiedlicher Motivation, vertreten Kendall Walton und Richard Wollheim. Für Walton (ders. 1990) sind die 
Sätze innerhalb von Make-Believe Spielen wahr. Für Wollheim (1980) dagegen können wir im Fall von Bildern eben in-Bildern-sehen, dass Andy eine platinfarbene Perücke trägt und dies ist ein Grund dafür, dass Satz (1) wahr sein kann, genauso wie gewöhnlicher Weise unser Sehen, dass Andy eine platinfarbene Perücke trägt, ein Grund dafür sein kann, dass Satz (1) wahr ist.

Gegen die Auffassung, dass Sätze der obigen Art, geäußert vor entsprechenden Bildern, wahre oder korrekte Äußerungen sein können, lässt sich einwenden, dass diese Sätze vor Bildern geäußert schlichtweg falsch oder unsinnig sind und man die Sache nur dadurch retten könne, dass man diese Sätze elliptisch auffasse. Für die obigen Sätze heißt dies, dass man sie beispielsweise folgendermaßen ergänzt:

(1.1) Auf dem Bild ist zu sehen, dass Andy eine platinfarbene Perücke trägt.

(2.2) Er sieht auf dem Bild Andy, der eine platinfarbene Perücke trägt.

Eine Schwierigkeit bei der elliptischen Auffassung ist, wie man die jeweilige Ergänzung legitimiert, d. h. genau diese und keine andere wählt. So lässt sich der Satz (1) offensichtlich je nach bildphilosophischer Auffassung auf vielfältige Weise ergänzen.

(1.2) Das Bild zeigt Andy, der eine platinfarbene Perücke trägt.

(1.3) Das Bild verursacht die Vorstellung, Andy mit einer platinfarbenen Perücke zu sehen.

$\left(1.3^{*}\right)$ Das Bild verursacht die Illusion, Andy mit einer platinfarbenen Perücke zu sehen.

(1.4) Das Bild ist von der Art Andi-trägt-eine-goldene-Perücke-Bild.

(1.5) In dem Bild ist Andy zu sehen, wie er eine platinfarbene Perücke trägt.

Ergänzungen der Art (1.1)-(1.3) werden vermutlich Ähnlichkeitstheoretiker geben, eine der Art (1.4) eventuell Goodmanianer und (1.5) von Wollheim inspirierte Theoretiker.

Eine andere Variante zur Lösung ist die Kontextauffassung. Sie besagt, dass die Sätze jeweils nur wahr in bestimmten Kontexten sind. Diese Auffassung hat offensichtlich Ähnlichkeit mit derjenigen Waltons: Wir sprechen eben anstatt von fiktionaler Wahrheit von wahr im Kontext F. So ist es in dem 
einen Kontext wahr, dass Andy eine platinfarbene Perücke trägt, und in dem anderen, dass Sherlock Holmes in London lebt.

Wählt man eine Lösung dieser Art, dann scheint es nahe zu liegen den Beispielsatz:

\section{(A) Achill hat einen goldenen Schild}

egal, ob bezogen auf ein Bild oder einen Roman, auf dieselbe Weise zu interpretieren, nämlich, dass es jeweils einen Kontext $\mathrm{F}$ gibt, in dem es wahr ist, dass auf Achill das Prädikat „hat einen goldenen Schild“ zutrifft. Ein Unterschied besteht (alleine) in der Verifikation des Satzes. Im Fall des Textes kann ich hierzu auf Textstellen verweisen, während ich beim Bild auf einen Teil des Bildes zeigen kann. Der Unterschied scheint also darin zu bestehen, dass ich mich im Fall der goldenen-Schild-Darstellung auf einen Gegenstand in der Welt, den ich sehe, beziehe, während ich mich im Fall der Ilias damit nur auf einen fiktionalen Gegenstand beziehe? An dieser Stelle müssen wir äußerst achtsam sein, um nicht wieder in eine Form der Ähnlichkeitstheorie zurückzufallen. Warum? Weil eine naheliegende Antwort lauten könnte, dass ich den goldenen Schild im Bild sehe, weil ich im Bild eine zweidimensionale Fläche sehe, die ähnlich zum dargestellten Gegenstand ist. Doch ob hier von einem gesehenen Gegenstand gesprochen werden kann, ist fraglich, wenn man sich an Wollheims Lokalitätsbedingung erinnert (diesen Punkt werde ich weiter unten noch genauer erörtern). Wir können zwar in Bildern auf etwas zeigen, aber daraus folgt nicht, dass das, worauf wir zeigen, ein Gegenstand ist, der dem dargestellten Gegenstand ähnlich ist. Zwei Punkte sind hier zu beachten:

Erstens, worauf wir zeigen, ist der „Bildgegenstand“, aber dieser muss kein physikalisch klar lokalisierbarer Gegenstand sein.

Zweitens, selbst wenn er klar von seiner Umgebung abgrenzbar ist, folgt daraus nicht, dass er die von der Ähnlichkeitstheorie bestimmten Eigenschaften mit dem dargestellten Gegenstand teilen muss. Der gemalte Schild muss etwa weder golden sein, noch die Umrissform mit dem Schild teilen. So trifft etwa das Prädikat ,ist golden“ auf keinen Teil des Bildes bzw. den Bildträger zu, wenn auch im Bildkontext gilt, dass der Ausdruck „,ist golden“ auf den Schild Achills zutrifft.

Aber, dass wir in Bildern auf Bildgegenstände zeigen können (und nicht nur auf Farbflecken etc.), lässt sich natürlich nicht leugnen. Ein Grund hier- 
für ist, dass in Bildern die Bildgegenstände räumlich angeordnet sind. Diese räumliche Anordnung ist für darstellende Bilder wesentlich und bestimmt unser Sehen der Bilder. Aber - und schon wieder grüßt der Ähnlichkeitstheoretiker - muss diese Anordnung der Bildgegenstände nicht ähnlich zu der Anordnung der Dinge in der Welt sein (zumindest bei nicht fiktiven Bildern)? Nein, wesentlich ist nur, dass wir vor dem Bild auf ähnliche Weise sprechen können wie vor der dargestellten Szene (und genau dies ist häufig der Zweck von Modellen). So kann ich beispielsweise, vor einem Bild stehend, auf eine Walddarstellung zeigen und sagen: „Dahinter liegt das Meer.“ Gleiches geht bei Texten nicht. Texte sind in diesem Sinne zweidimensional, d.h. die Ordnung ist durch die Linearität des Lesens geprägt. Wir können zwar auch bei Texten sagen, dass steht weiter hinten, aber „hinten“ wird bestimmt durch die Linearität des Textes. Erst recht gilt dies für das Zeigen. Ich kann immer nur auf die Wörter im Text zeigen, aber dabei zeige ich nicht auf ihre Bedeutung, so wie ich bei einem Bild auf einen gelben Farbfleck zeigen kann und dabei sage, schau dir den goldenen Schild an. Bilder oder besser gesagt: der Bildraum ist im Gegensatz zu Texten dreidimensional. Die Orientierung in einem Text ist in diesem Sinne grundverschieden von der Orientierung in einem Bild. Eine weitere Frage ist, auf welche Weise sich die Orientierung nach einem Text von der Orientierung nach einem Bild unterscheidet. Man denke beispielsweise an bildliche Gebrauchsanweisungen. In diesen können räumlich Situationen (etwa wie die Finger zu halten sind) präziser und vor allen Dingen einfacher und klarer dargestellt werden, als wenn man sie beschreiben wollte.

Die entscheidende Frage ist gleichwohl, was unser Tun (das Zeigen, das Sprechen etc.) vor Bildern legitimiert. Nach Walton sind es die Spielregeln des Tun-als-ob Spiels. Aber - und hier scheint die Schwäche Waltons zu liegen wir tun doch nicht nur so, als ob wir den Schild von Achill sehen, wir sehen den Schild Achills im Bild! Wollheim betont daher auch immer wieder, dass hier die Stärken seines Ansatzes liegen und das Sehen-in kein Imaginieren ist, sondern eine spezielle Wahrnehmungsform des Sehens (vgl. Wollheim 2001, S. 24 f). Der Punkt, auf den Wollheim hier hinweist, scheint tatsächlich nur in seiner Theorie genügend berücksichtigt zu werden und lässt sich nur schwer, wenn überhaupt, in die Ansätze von Walton aber auch Goodman einarbeiten. Letzterer kommt scheinbar in Schwierigkeiten, wenn er ein Bild Achills mit seinem goldenen Schild analog zu einem sprachlichen Ausdruck auffasst, dem wir gewissermaßen eben nicht ansehen, was er bezeichnet. 
Betrachten wir nun dazu Wollheims Charakterisierung des Sehens-in, um vor dem Hintergrund dieser Konzeption auf unsere Frage zurückzukommen, was unser Tun (das Zeigen, das Sprechen etc.) vor Bildern legitimiert.

Wollheims Sehen-in lässt sich wie folgt charakterisieren:

i. Was wir in einem Bild korrekterweise sehen, muss vom Bildhersteller intendiert sein.

ii. Sehen-in im Gegensatz zum Sehen-als umfasst auch das Sehen von Ereignissen und nicht nur von Einzeldingen (vgl.Wollheim 1980, S. 210).

iii. Während wir beim Sehen-als das Gesehene immer lokalisieren können (beispielsweise können wir die Beine des Schafs in der Wolke lokalisieren), ist Gleiches beim Sehen-in nicht möglich. Wir können z. B. bei der Dornenkrönung Tizians nicht auf die Stelle deuten, an der Christus gequält wird (ebd. S. 211).

iv. Sehen-in erfordert Zweiheitssehen, d. h. zum einen das Sehen des Bildträgers und zum anderen das Sehen des Bildinhalts (ebd. S. 212 ff.).

Akzeptiert man Wollheims vier Bedingungen, hat man eine erste Antwort darauf bekommen, in welcher Hinsicht sich der Kontext zwischen normaler Sprachverwendung und dem Kontext Reden über Bilder unterscheidet.

So können die Sätze (1) bis (12) für Wollheim angesichts von Bildern nur wahr sein, wenn sie den Intentionen des Produzenten gerecht werden. Diese Forderung halte ich für zu stark, ohne an dieser Stelle dafür argumentieren zu wollen. (So scheint es mir fraglich, ob wir uns auch nur einen Fall vorstellen können, in dem mein Sehen-in alleine (!) deshalb falsch ist, weil es den Intentionen des Betrachters widerspricht.) Wollheims zweite Bedingung ist vor allen Dingen dafür gedacht, das Sehen-in vom Sehen-als zu unterscheiden. Sie soll uns hier auch nicht weiter interessieren. Die dritte Bedingung ist insofern wichtig, als sie andeutet, dass Ähnlichkeitstheorien mit Wollheims Auffassung unvereinbar sind und sich das Zeigen von „Bildgegenständen“ vom Zeigen von gewöhnlichen Gegenständen unterscheidet. Letztere sind üblicherweise klar diskriminierbare Gegenstände, die sich von ihrer Umgebung abheben. Ein Grund hierfür ist, dass wir meistens um sie herumlaufen können etc. Die vierte Bedingung, das zweiheitliche Sehen, ist dagegen zentral. Es gehört eben zu den Wahrheitsbedingungen des Sehen-in, dass wenn jemand z. B. in einem Bild Andy Warhol sieht, er auch gleichzeitig den Bildträger sieht und daher 
weiß, dass er nicht Andy Warhol in Natura sieht. D. h. aus dem im Bildkontext geäußerten Satz (1) folgt, dass auch ein Bildträger zu sehen ist. Präziser: Wenn Satz (1) im Bildkontext wahr ist, dann ist

Es existiert ein Bildträger.

oder

Der Sprecher von (1) sieht einen Bildträger.

im gewöhnlichen Kontext wahr.

Dieser Kontextwechsel in den Folgerungsbeziehungen geht also mit einem Wechsel der Kategorien I-IV einher. Wir folgern eben aus einem Satz der Kategorie I auf Sätze der Kategorien II-IV und verlassen somit den fiktionalen Bildkontext.

Wendet man sich diesen Kontextwechseln zu, stellt man sogleich fest, dass hier keine Ähnlichkeiten zwischen unserer Rede über Bilder und unserer gewöhnlichen Rede vorliegen, weil beispielsweise dann, wenn wir Satz (1) im gewöhnlichen Kontext äußern, Folgerungen dieser Art nicht erlaubt sind. Paradoxerweise sind aber Ähnlichkeitstheoretiker genau an diesen Folgerungen zumeist interessiert. So würde für einen Umrisstheoretiker wie Hyman aus (1) folgen, dass Andy, soweit dieser dann existiert und von einem bestimmten Blickwinkel aus betrachtet wird, die gleiche Umrisslinie wie der Bild-Andy (die Farbfläche auf der Leinwand, die Andy darstellt) hat. Eine Folgerung dieser Art ist aber weder logisch notwendig noch wahr, sondern in vielen Fällen einfach schlichtweg falsch. Genau so wenig folgt aus (1), dass auf dem Bildträger platinfarbene Flächen zu sehen sein müssen. Der springende Punkt ist vielmehr der, den auch alle Antiähnlichkeitstheoretiker teilen, dass aus Sätze wie (1) im Bildkontext geäußert überhaupt keine Folgerungen - außer dass ein Bildträger vorliegen muss - auf Sätze außerhalb des Bildkontextes vorliegen müssen. D. h. ein Bild in dem (1) zu sehen ist, kann ein schwarz weiß Bild, ein Farbnegativ oder was sonst auch immer sein.

Was alleine aus Sätzen wie (1) oder (2) im Bildkontext folgt, sind Folgerungen der Art wie

Da ist eine platinfarbene Perücke

oder

Er sieht eine platinfarbene Perücke. 
Die Sache ist in dieser Hinsicht vergleichbar mit Wittgensteins Analyse von Vorstellungen. Daraus, dass einer eine Vorstellung hat, wie Andy eine platinfarbene Perücke trägt, folgt bezogen auf eine mögliche Realisierung der Vorstellung nicht, dass meine Hirnstruktur oder Ähnliches notwendigerweise eine bestimmte Struktur besitzen muss. Dagegen folgt, wenn jemand sich Andy mit einer platinfarbenen Perücke vorstellt, dass er sich auch eine platinfarbene Perücke, einen Menschen, ein Lebewesen etc. vorstellt. Zudem gilt, wenn er sich (1) visuell vorstellt, dass er sich keinen Glatzkopf vorstellt. Dies trifft auch dann zu, wenn er weiß, dass Andy in Wirklichkeit ein Glatzkopf ist (vgl. zur Logik von visuellen Vorstellungen Steinbrenner 2006). Gleiches gilt in dieser Hinsicht für Bilder bzw. unsere Beschreibungen von Bildern, auch wenn sie sich natürlich grundlegend von Vorstellungen bzw. unseren Beschreibungen von Vorstellungen unterscheiden. Denn nur auf Bilder können wir zeigen und haben für ihre materiellen Gegenstücke intersubjektiv gültige Interpretationsregeln, sprich angelernte Verhaltensdispositionen, die es uns ermöglichen, diese als Bilder zu verwenden, d. h. in ihnen etwas zu sehen. Diese Dispositionen sind Grund dafür, dass wir gemeinsam Bilder betrachten können und uns darüber einig sind, was auf ihnen zu sehen ist. Diese Einigkeit drückt sich im Wesentlichen darin aus, dass wir wissen, welche Sätze bezogen auf das Bild wahr sind. So trifft im Kontext der Ausdruck „platinfarben“ auf Andys Perücke zu, ganz unabhängig davon, ob dieser Ausdruck im gewöhnlichen Kontext auf den Bildträger zutrifft. Um Missverständnisse auszuschließen: Es gibt natürlich genau so wenig nur einen Bildkontext wie es auch nicht nur eine Sprache gibt. D. h., so wie derselbe Ausdruck je nach Sprache falsch oder wahr sein kann, so kann derselbe Ausdruck innerhalb zweier Bildkontexte einmal falsch und einmal wahr sein.

Wenn ich auch mit Walton und Hyman der Meinung bin, dass die fiktionale Verwendung von Bildern der abbildenden vorausgeht, darf man natürlich nicht unterschätzen, inwieweit der abbildende Gebrauch Auswirkungen auf den fiktionalen hat. Das heißt durch die Zentralperspektive und insbesondere dann später durch die Fotografie kommen Interpretationsregeln (aufgrund von Projektionsregeln) ins Spiel, die unsere Redeweise über Bilder verändern. Man denke nur an die Behauptung (vgl. Hertz 1884, S. 112 ff.), dass noch der kleinste Punkt auf einem Gemälde repräsentational sei. Dies trifft zwar nicht einmal auf Fotografien zu, aber trotzdem hat dieser Gedanke Auswirkungen auf unsere Rede über Bilder. So können wir bei jeder größeren Fläche eines Bildes, die nicht eindeutig funktioniert, beginnen, über mögliche Bildgegenstände zu 
spekulieren. Beispielsweise können wir uns Gedanken darüber machen, ob der Hintergrund eines Bildes als eine Wand oder als Himmel zu sehen ist. Diese Spekulationen sind aber nicht wahllos, sondern werden immer von den vorhergehenden Interpretationen abhängen bzw. sollen mit ihnen vereinbar sein. Man denke bspw. auch an Glanzlichter. Dass ich das Weiß auf einem dargestellten dunklen Stoff nicht als weißen Fleck auf dem Stoff sehe, sondern als Reflex, hängt von der allgemeinen Lichtführung im Bild ab.

Wenn auch der Gedanke, dass jeder noch so kleine Fleck auf dem Bild für etwas steht, falsch ist, gilt gleichwohl für Bilder wie für ihre realen Gegenstücke, dass sie es uns ebenso erlauben, ins Detail zu gehen und so Neues zu entdecken, wie auch durch den Gewinn von Abstand allgemeinere Strukturen zu erkennen.

Aber, so könnte hier der Ähnlichkeitstheoretiker wieder einwenden, zeigt dies nicht gerade, dass Bilder ihren realen Gegenstücken, falls vorhanden, ähnlich sind? Ja, aber die Ähnlichkeit zeigt sich nicht in (!) den Bildern, sondern in unserem Verhalten vor ihnen und ihren möglichen realen Gegentücken. Denken wir hierzu an Gombrichs bekanntes Steckenpferd. Der Pferdekopf ist die Bürste und das Kind reitet auf dem Stiel. Wird man nun das Kind fragen, ob das Pferd auch mit seinem Schweif wedeln kann, wird das Kind wahrscheinlich mit dem Stielende wackeln. Das heißt, es werden bestimmte räumliche oder auch andere Merkmale, die auf echte Pferde zutreffen, versuchsweise auf den Besen übertragen. Manche von ihnen werden eindeutig sein (Pferdekopf und Rücken), andere werden sich erst im Laufe des Spiels entwickeln. Inwieweit diese Übertragungsregeln explizit oder implizit gelten, ist eine Frage, die uns hier nicht beschäftigen braucht. Wichtig ist nur, dass wir diese Regeln irgendwann so internalisiert haben, dass wir unwillkürlich im Bild dies und das sehen. Eine Folge hiervon ist, dass wir gegenüber Bildern erst einmal so tun - so sprechen, darauf deuten usw. - wie im Falle der Gegenstände selbst. Voraussetzung dafür ist, dass wir Bilder in gewisser Hinsicht ähnlich klassifizieren wie gewöhnliche Gegenstände. Wir sehen beispielsweise im Bild einen goldenen Schild, das heißt wir können das Bild als goldenes-Schild-Bild klassifizieren. Das bedeutet, wenn jemand im Bild einen goldenen Schild sieht, dann folgt daraus, dass er den Schild als goldene-Schild-Darstellung klassifizieren kann. Die Klassifikation unterscheidet sich dabei nicht grundsätzlich von der, einen Schild als golden zu klassifizieren. Das heißt aber auch, dass die Eigenschaft, ein goldenes-Schild-Bild zu sein, sich im nominalistischen Sinn auf die gleiche 
Weise erklären lässt, wie die Eigenschaft ein goldener Gegenstand zu sein. Das heißt, wir brauchen keine Eigenschaften oder Essenzen anzunehmen, die Grund dafür sind, dass der Schild golden und das Bild eine goldeneSchild-Darstellung ist, sondern nur auf unseren Sprachgebrauch achten, in dem eben gegebenenfalls der Schild als ,golden“ und das Bild als ,goldeneSchild-Darstellung“ bezeichnet wird. Fragt nun jemand, warum dem so sei, sollte man sich an Wittgenstein Antwort auf die Frage erinnern:

Wie erkenne ich, dass diese Farbe Rot ist? - Eine Antwort wäre: „Ich habe Deutsch gelernt.“(Wittgenstein 1982, § 383)

\section{Literatur}

Arthur Danto, The Transfiguration of the Commonplace. A Philosophy of Arts (Harvard University Press, 1981).

Heinrich Hertz, Die Prinzipien der Mechanik (Leipzig, 1884).

John Hyman, The Objective Eye: Color, Form, and Reality in the Theory of Art (University of Chicago Press, 2006).

Dominic Lopes, Understanding Pictures (Oxford, 1996).

Oliver R. Scholz, Bild, Darstellung, Zeichen: Philosophische Theorien bildlicher Darstellung (Frankfurt, 2004).

Jakob Steinbrenner, „Die Ähnlichkeit und die Bilder“ (1998), in K. Rehkämper / K. Sachs-Hombach: Bild, Bildwahrnehmung, Bildverarbeitung: Interdisziplinäre Beiträge zur Bildwissenschaft, (Wiesbaden: Deutscher Universitätsverlag, 1998), 125-131.

Jakob Steinbrenner, „Bilder von Vorstellungen und Vorstellungen von Bildern: Zur Kritik Wittgensteins an Freges Vorstellungsbegriff“ (2006), Deutsche Zeitschrift für Philosophie 6 (2006): 907-924.

Jakob Steinbrenner, „Analytische Bildtheorien“, in K. Sachs Hombach: Bildtheorie, (Frankfurt a. M.: Suhrkamp (stw), 2009), 284-315.

Jakob Steinbrenner, „Was heißt Bildkompetenz? - Oder Bemerkungen zu Dominic Lopes' Kompetenzbedingung“, in M. Greenlee u.a.: Aisthesis, (Regensburg: Schnell \& Steiner, im Erscheinen), 57-69.

Kendall L. Walton, Mimesis as Make-Believe: On the Foundations of the Representional Arts (Cambridge Mass: Harvard University Press, 1990).

Ludwig Wittgenstein, Philosophische Untersuchungen (Frankfurt, 1982).

Richard Wollheim, „Seeing-as, Seeing-in, and Pictorial Representation“, in Richard Wollheim: Art and its Objects (Cambridge University Press 1980), 205-226. 
Richard Wollheim, „On Pictorial Representation“, in Rob van Gerwen: Richard Wollheim on the Art of Painting: Art as Representation and Expression, (Cambridge: University Press, 2001), 13-27. 